
\title{
CIÊNCIA'NATURA
}

\section{Nova Abordagem para a Estimativa do Fluxo de Calor no Solo para o Bioma Pampa}

\author{
New Approach for the Estimation of Soil Heat Flux for the Pampa Biome
}

\author{
${ }^{1}$ Leugim Corteze Romio, ${ }^{2}$ Tamíres Zimmer, ${ }^{2}$ Débora Regina Roberti e ${ }^{2}$ Lidiane Buligon \\ ${ }^{1}$ Universidade Federal do Pampa (UNIPAMPA) - Campus Itaqui-RS , Brasil \\ ${ }^{2}$ Universidade Federal de Santa Maria - UFSM, Brasil
}

\begin{abstract}
Resumo
O estudo das propriedades térmicas do solo e das variáveis dependentes destas propriedades é de suma importância para a compreensão do balanço de energia da superfície. Dentre as principais variáveis há o fluxo de calor no solo, especialmente, por permitir a verificação das influências que ocorrem no bioma de uma região à medida que ocorrem variações de temperatura. Este trabalho tem como objetivo estimar o coeficiente de condutividade térmica e, consequentemente, obter os valores para o fluxo de calor no solo. Os dados utilizados para estimação dos parâmetros foram obtidos de um sítio experimental localizado no município de Pedras Altas-RS. As simulações foram realizadas a partir de duas equações, a primeira definida pela Lei de Fourier da Condução de Calor (discretizada) clássica e a segunda pela modificação da primeira incluindo um coeficiente $k$, denominado "erro de modelagem". Com base nos resultados obtidos pode-se verificar que a equação modificada apresentou melhores resultados, quando comparada a solução clássica.
\end{abstract}

Palavras-chave: Fluxo de Calor no Solo, Bioma Pampa, Balanço de Energia da Superfície.

\section{Abstract}

The study of the thermal properties of the soil and the variables dependent on these properties is very important for the understanding of the surface energy balance. Among the main variables, there is the soil heat flux, especially because it allows the verification of the influences that occur in the biome of a region as temperature variation occurs. This work intend to estimate the coefficient of thermal conductivity and, thus, to obtain the values for the soil heat flux. The data used to estimate the parameters were obtained from an experimental site located in the city of Pedras Altas-RS. Two equations were used, the first one defined by the Fourier Heat Conduction Law (discretized) and the second one a modification of the first one including a $k$ coefficient, named "modeling error". Based on the results obtained it can be verified that the modified equation presented better results, when compared with the classical solution.

Keywords: Soil Heat Flux, Pampa Biome, Surface Energy Balance. 


\section{Introdução}

A análise das propriedades térmicas do solo é de significativa importância para o estudo do balanço de energia da superfície, pois, a partir delas é possível estimar, com maior acurácia, a temperatura e o fluxo de calor no solo.

As principais propriedades são condutividade térmica, a qual pode ser caracterizada como a medida da capacidade de conduzir calor (Oke, 1987), difusividade térmica, a qual pode ser descrita como a capacidade de difundir as influências térmicas (Oke, 1987) e capacidade calorífica volumétrica, que pode ser definida como a quantidade de calor necessária para aumentar uma unidade de volume $\left(\mathrm{m}^{3}\right)$ de uma substância através de uma variação de 1 grau $(K)$ na temperatura (Oke, 1987). Estas propriedades, em geral, variam com a temperatura, e indiretamente, com a umidade do solo, a composição do solo, a estrutura física do solo e a cobertura de vegetação.

Dentre as variáveis que dependem das propriedades térmicas do solo, há o fluxo de calor no solo, o qual é considerado um dos importantes parâmetros no estudo do balanço de energia da superfície. Pois, permite verificar as influências que ocorrem no bioma de uma região à medida que ocorrem variações de temperatura. Por exemplo, compreender os diferentes ciclos da flora de um determinado bioma definido por diferentes estações do ano e, qual a relação existente com a variação de temperatura do solo em cada estação. Além disso, pesquisas têm indicado que o fluxo de calor no solo está relacionado a evapotranspiração, a exemplo, a de Kustas e Daughtry (1990).

Liebethal e Foken (2007) realizaram um resgate histórico das principais abordagens utilizadas para estimativa do fluxo de calor no solo, destacando seis (6) como sendo as mais utilizadas. Entretanto, os principais modelos estimam essa variável com base no balanço de radiação.

Outra forma de obter o fluxo de calor no solo é por medição direta utilizando sensores. Entretanto, sua medição requer a instalação destes sensores em diferentes profundidades. Bem como, a medição conjunta das temperaturas nestas profundidades de modo que seja possível calcular o "calor" armazenado (An et al., 2016; Russel et al., 2015). Além disso, os dados coletados por meio de experimentos de campo não descrevem diretamente as propriedades térmicas do solo. Neste caso, uma abordagem alternativa (proposta neste trabalho) é, com o auxílio de modelos escritos em função da variação de temperatura, estimar o coeficiente de condutividade térmica $(\lambda)$ e a partir deste obter o fluxo de calor no solo $\left(G_{z m}\right)$ que, consequentemente, permitirá calcular, também, a capacidade térmica volumétrica.

Neste contexto, este trabalho estima o coeficiente de condutividade térmica a partir de uma modelagem numérica e de dados coletados em um sítio experimental localizado na região do Bioma Pampa. Aqui propõe-se o acréscimo de um termo independente na Lei de Fourier da Condução de Calor (discretizada) clássica que representa o que denominamos de "erro de modelagem".

\section{Materiais e Métodos}

\subsection{Sítio Experimental}

As amostras relacionadas ao fluxo de calor no solo e temperatura no solo foram coletadas em um sítio experimental localizado no município de Pedras Altas ( $31^{\circ} 43^{\prime} 56^{\prime}$ ' $S$; $53^{\circ} 32$ ' 36” $\mathrm{W}, 395 \mathrm{~m}$ ) no interior do estado do Rio Grande do Sul (Brasil). A região possui solo Franco Arenoso, predominantemente raso, classificado como Neossolo e Cambissolo (da Cunha et al., 2005) e vegetação predominantemente de "pastagem". Para este trabalho, foram utilizados dados referentes ao mês de Julho de 2015.

Os sensores utilizados para medição da temperatura foram $T 108$ (Campbell Sci), posicionados a 0,05 $m$ e a $0,15 \mathrm{~m}$ abaixo da superfície e, para o fluxo de calor no solo foi HFP01 (Hukseflux), posicionado a $0,10 \mathrm{~m}$, de modo que este esteja na posição média entre as amostras de temperatura. 


\subsection{Modelo Proposto para o Fluxo de Calor no Solo}

O fluxo de calor no solo a uma profundidade $z$ é descrito pela Lei de Fourier da Condução de Calor (Carslaw e Jaeger, 1959), cujo valor do fluxo é dado por:

$$
G_{z m}=-\lambda \frac{\partial T}{\partial z}
$$

sendo que $G_{z m}$ é o fluxo de calor no solo (em $\left.W m^{-2}\right)$ a uma profundidade $z, \lambda$ é a condutividade térmica $\left(W m^{-1} K^{-1}\right), T$ é a temperatura do solo (em $K$ ) e $z$ é a profundidade abaixo da superfície do solo (em $m$ ).

Com base no processo de amostragem, os dados obtidos experimentalmente estão discretizados, sendo assim, considera-se a aproximação para a taxa de variação da temperatura em função da profundidade dada pela a Equação 2:

$$
\frac{\partial T}{\partial z} \approx \frac{T_{2}-T_{1}}{z_{2}-z_{1}}
$$

em que $T_{1}$ e $T_{2}$ são as temperaturas nas profundidades $z_{1}$ e $z_{2}$, respectivamente. Supondo que $\lambda$ seja independente da profundidade (An et al., 2016; Carslaw e Jaeger, 1959), a Equação 1 pode ser escrita da seguinte forma:

$$
G_{z m}=-\lambda \frac{T_{2}-T_{1}}{z_{2}-z_{1}}
$$

A aproximação calculada pela Equação 3, em geral, leva em consideração a temperatura a nível de superfície $(z=0 \mathrm{~m})$ como sendo uma condição de contorno. Entretanto, para este problema não é apropriado considerar condições de contorno homogêneas. Russel et al. (2015) mencionam que, em geral, o fluxo de calor no solo é frequentemente medido a uma certa profundidade (z) abaixo da superfície e que deve-se levar em consideração a quantidade de energia "armazenada" entre a superfície e a profundidade de amostragem. De modo que, para representar esta situação, o fluxo de calor na superfície do solo é, então, descrito pela soma de $G_{z m}$ a quantidade de energia "armazenada" $\left(S_{G}\right)$ entre a superfície e a profundidade de amostragem, ficando assim definida:

$$
G_{0}=G_{z m}+S_{G}
$$

Por fim, a Equação 4, combinada a Equação 3, passa a ser descrita matematicamente por:

$$
G_{0}=\lambda \frac{T_{2}-T_{1}}{z_{2}-z_{1}}+S_{G}
$$

Entretanto, $G_{z m}$ deve levar em consideração os possíveis "erros de modelagem". Neste sentido, propõe-se o acréscimo de um termo $(k)$, inicialmente constante (em particular, independente da profundidade), para estimativa do fluxo de calor no solo a uma profundidade $z$. De modo que a equação 1 passa a ser definida por:

$$
G_{z m_{1}}=-\lambda_{1} \frac{\partial T}{\partial z}+k
$$

Levando em consideração dados discretizados, conforme já mencionado, a equação passa a ser escrita por:

$$
G_{z m_{1}}=-\lambda_{1} \frac{T_{2}-T_{1}}{z_{2}-z_{1}}+k
$$

E, o cálculo do fluxo de calor na superfície do solo pode, então, ser calculado por:

$$
\begin{aligned}
G_{0} & =G_{z m_{1}}+S_{G} \\
& =-\lambda_{1} \frac{T_{2}-T_{1}}{z_{2}-z_{1}}+k+S_{G}
\end{aligned}
$$


Com base no exposto, este artigo apresenta, os resultados relativos ao modelo sugerido para o fluxo de calor no solo a uma profundidade $z$, descrito pela Equação 7 .

\section{Resultados}

A equação 3 foi utilizada para estimar o valor de $\lambda$ e a Equação 7 para estimar os valores de $\lambda_{1}$ e $k$. Os ajustes foram obtidos pelo Método dos Mínimos Quadrados, utilizando os dados experimentais descritos na Seção 2.

Para o caso simulado pela Equação 3, o valor de $\lambda$ obtido foi de $1,1434 \mathrm{~W} \mathrm{~m}^{-1} \mathrm{~K}^{-1}$ e o erro quadrático médio, entre os valores do fluxo de calor no solo experimental e estimado $\left(G_{z m}\right)$, da ordem de 5,647 .

A Figura 1 apresenta a comparação entre os dados experimentais e os valores estimados do fluxo de calor do solo considerando a Equação 3.

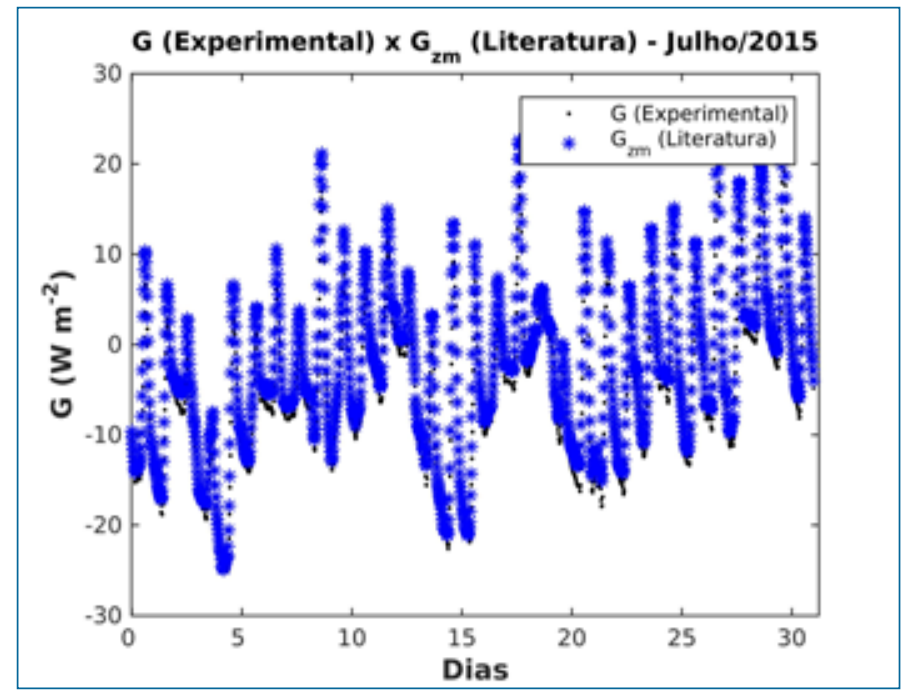

Figura 1: Fluxo de calor no solo experimental e simulado pela Equação 3.

A Figura 2 ilustra a comparação entre os dados experimentais e os valores estimados para o fluxo de calor do solo simulado pela Equação 7. Nesta simulação os valores de $\lambda_{1}$ e $k$ estimados foram, respectivamente, $1,0774 W^{-1} \mathrm{~K}^{-1}$ e $2,1256 W^{-2}$, e o erro quadrático médio, entre os valores do fluxo de calor no solo experimental e proposto $\left(G_{z m_{1}}\right)$ da ordem de 1,4343 .

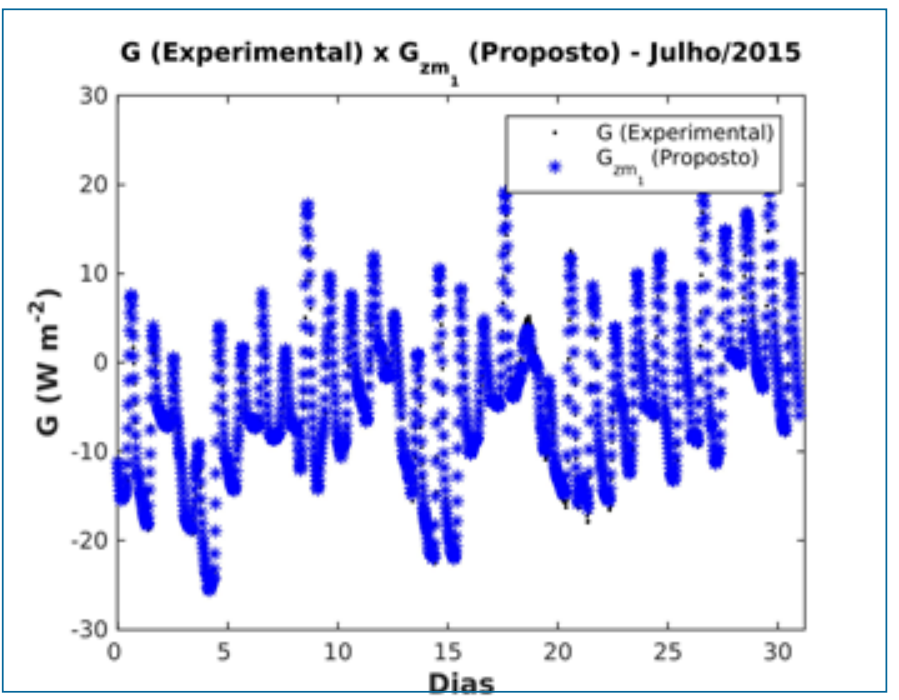

Figura 2: Fluxo de calor no solo experimental e estimado pela Equação 5. 
Observa-se que os resultados obtidos nesta segunda simulação são, consideravelmente, melhores que os apresentados na primeira. Estes resultados comprovam a hipótese de que existe o "erro de modelagem”, aqui proposto. Um dos pontos a destacar está relacionado ao fato de os períodos noturnos serem melhor representados pelo modelo proposto na Equação 7.

A Figura 3 apresenta o gráfico de dispersão entre os valores do fluxo de calor no solo experimental e o fluxo de calor no solo estimado pela Equação 7. Novamente os resultados simulados estão de acordo com os experimentais.

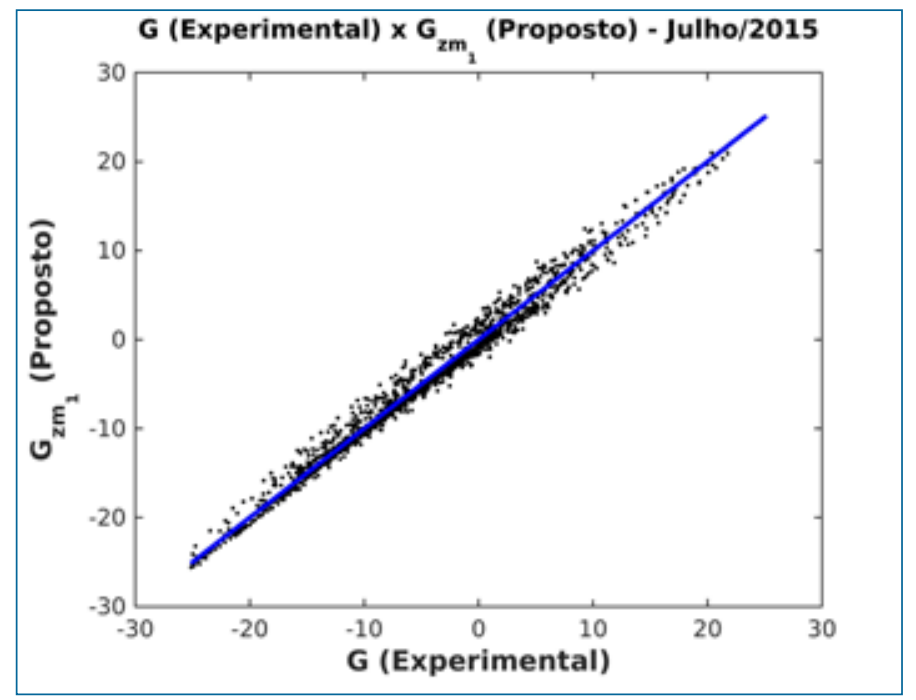

Figura 3: Gráfico de dispersão entre os dados experimentais e os estimados pela Equação 7.

\section{Conclusões}

O presente trabalho propõe um novo modelo numérico para estimar o coeficiente de condutividade térmica e, consequentemente, o fluxo de calor no solo, supondo a existência de "erro de modelagem". Os dados experimentais de temperatura e de fluxo de calor no solo foram coletados no município de Pedras Altas, no interior do estado do Rio Grande do Sul, situado na região do bioma Pampa.

As simulações foram realizadas levando em consideração duas equações, sendo a primeira a clássica encontrada na literatura (Equação 3) e a segunda, que consiste em uma modificação da Equação 3 pelo acréscimo de um termo que representa o "erro de modelagem" (Equação 7).

Com base nos resultados apresentados (Figuras 1 e 2), pode-se concluir que é necessário levar em consideração o "erro de modelagem" e que este influencia significativamente na estimativa do fluxo de calor no solo.

É importante destacar que este é um trabalho inicial, outros testes estão sendo realizados, verificando as possíveis relações do coeficiente $k$ com outras propriedades/variáveis térmicas. Além de trabalhos, utilizando a Equação 8, para estimativa do fluxo de calor na superfície do solo, valor importante para o cálculo do balanço de energia na superfície do solo.

\section{Referências}

An, K., Wang, W., Zhao, Y., Huang, W., Chen, L., Zhang, Z., Wang, Q., Li, W. (2016). Estimation from soil temperature of soil thermal diffusivity and heat flux in sub-surface layers. Boundary-Layer Meteorology, 158, 473-488.

Carslaw, H. S., Jaeger, J. C. (1959). Conduction of Heat in Solids, $2^{\circ}$ edn. Oxford University Press, London.

da Cunha, N. G., da Costa Silveira, R. J., Severo, C. R. S., Mendes, R. G., da Silva, J. B., Duarte, L. R., Schumacher, R. L. (2005). Estudo dos Solos do Município de Pedras Altas-RS. Embrapa Clima Temperado, Pelotas.

Kustas, W. P., Daughtry, C. S. T. (1990). Estimation of the soil heat flux/net radiation ratio from spectral data. Agricultural and Forest Meteorology, 49, 205-223. 
Liebethal, C., Foken, T. (2007). Evaluation of six parameterization approaches for the ground heat flux. Theoretical and Applied Climatology, 88, 43-56.

Oke, T. R. (1987). Boundary Layer Climates, $2^{\circ}$ edn. Routledge, London.

Russel, E. S., Liu, H., Gao, Z., Finn, D., Lamb, B. (2015). Impacts of soil heat flux calculation methods on the surface energy balance closure. Agricultural and Forest Meteorology, 215, 189-200.

\section{Leugim Corteze Romio}

Tamíres Zimmer

Débora Regina Roberti Universidade Federal do Pampa (UNIPAMPA) - Campus Itaqui-RS , Brasil
E-mail: leugimcr@bol.com.br

Universidade Federal de Santa Maria - UFSM, Brasil

E-mail: tz.tamireszimmer@gmail.com

Universidade Federal de Santa Maria - UFSM, Brasil

E-mail: debora@ufsm.br

\section{Lidiane Buligon}

Universidade Federal de Santa Maria - UFSM , Brasil E-mail: prof.buligon@gmail.com 